\title{
Erratum: New Ground-State Crystal Structure of Elemental Boron [Phys. Rev. Lett. 117, 085501 (2016)]
}

\author{
Qi An, K. Madhav Reddy, Kelvin Y. Xie, Kevin J. Hemker, and William A. Goddard III \\ (Received 29 December 2016; published 12 April 2017)
}

DOI: 10.1103/PhysRevLett.118.159902

Our Letter reported both experimental and computation evidence for the new $\tau$ phase of elemental boron, which is closely related to the long known $\beta$ phase. Our experiments clearly established that both phases are present in high purity (99.2\%) samples of boron, with each grain being fully $\beta$ or fully $\tau$. Highly twinned structures have been observed previously [1] as have observations that twinning decreases as the high purity $\beta$-B grows from the liquids [2]. However, our Letter focuses on identifying a new crystal phase that we discovered experimentally, $\tau$-B, determining its crystal structure that matches our experimental diffraction pattern and elucidating the nature of this new phase using DFT.

We also reported QM calculations that predicted the $\tau$ form to have a free energy at $0 \mathrm{~K}$ that is more stable than $\beta$. We now know this result to be incorrect. We thank Ogitsu et al. [3] for pointing out that the QM calculations we reported on the $\alpha-, \beta$-, and $\gamma$-B phases were carried out in 2013 using the standard projector-augmented wave pseudopotential available in VASP at that time [4-7]. However, our more recent calculations in 2016 of the $\tau$-B phases used the current pseudopotential that had been modified to allow for calculations with meta-GGA functionals [4-7].

We have now recalculated the various phases using the new pseudopotential with the Perdew-Burke-Ernzerhof (PBE) functional. Because the $\beta$ and $\tau$ phases have partial occupation of the various sites available in the space group, we considered the 10 cases summarized in Table I. The final result is that the lowest energy for the $\tau$ phase has an electronic energy $1.8 \mathrm{meV} / \mathrm{B}$ above the $\beta$ phase and $2.9 \mathrm{meV}$ above the alpha phase. Including the phonon corrections from the PBE calculations to correct for zero-point energy, the enthalpy at $0 \mathrm{~K}$ for $\tau$ with PBE is $1.7 \mathrm{meV} / \mathrm{B}$ higher than $\beta$ and $0.6 \mathrm{meV} / \mathrm{B}$ lower than $\alpha$-B. The lowest energy structure for $\tau-\mathrm{B}_{106}$ is displayed in Fig. 1.

Summarizing, our previous conclusion that $\tau$ - $\mathrm{B}_{106}$ is the most stable phase of elemental boron is clearly wrong. This difference of $1.7 \mathrm{meV} / \mathrm{B}$ enthalpy at $0 \mathrm{~K}$ in the $\beta$ and $\tau$ phases leads to twin interfacial energy for $\tau$ - $\mathrm{B}_{106}$ of $32.2 \mathrm{~mJ} / \mathrm{m}^{2}$.

We also used the phonon levels to calculate the entropy and Helmholtz free energy [9] as a function of temperature, leading to the free energy differences between $\beta-\mathrm{B}_{106}$ and $\tau-\mathrm{B}_{106}$ displayed in Fig. 2. The red dashed curve uses the phonon calculation for the 106 atom crystal unit cell $\beta$ - $\mathrm{B}_{106}$ and the 212 atom crystal unit cell $\tau$ - $\mathrm{B}_{106}$. This indicates that $\tau-\mathrm{B}_{106}$ may be more stable than $\beta-\mathrm{B}_{106}$ at temperatures above $950 \mathrm{~K}$, which may explain why many grains in boron have this structure. We believe that comparing the phonon contributions for systems with two different numbers of atoms per cell might bias the corrections because of the particular sampling of the Brillouin zone, so we recalculated the phonon corrections for $\beta$ - $\mathrm{B}_{106}$ using the doubled unit cell (212 atoms). This leads to the black line, indicating that the $\beta-\mathrm{B}_{106}$ phase is the most stable phase until the melting temperature of $2350 \mathrm{~K}$.

TABLE I. Electronic energy of 10 variations on the disordered B13 and B16 sites of the $\tau$ - $\mathrm{B}_{106}$ structure, compared with $\beta$-B 106 and $\alpha$-B. The zero-point energy corrections $(\mathrm{meV} / \mathrm{B})$ are $126.74\left(\tau-\mathrm{B}_{106}\right), 126.83\left[\beta-\mathrm{B}_{106}\right.$, and $\left.130.02(\alpha-\mathrm{B})\right]$, while the free energy corrections $(\mathrm{meV} / \mathrm{B})$ from 0 to $298 \mathrm{~K}$ are $-5.364\left(\tau-\mathrm{B}_{106}\right),-5.356\left(\beta-\mathrm{B}_{106}\right)$, and $-4.545\left(\alpha-\mathrm{B}_{12}\right)$. The specific locations of all atoms for these 10 structures are available in Ref. [8].

\begin{tabular}{lccc}
\hline \hline$\tau$ - $\mathrm{B}_{106}$ structures & Energy $(\mathrm{eV} / \mathrm{B})$ & $\tau$ - $\mathrm{B}_{106}$ structures & Energy $(\mathrm{eV} / \mathrm{B})$ \\
\hline S-1 (Ref. [4]) & -6.6918 & $\mathrm{~S}-6$ & -6.6932 \\
S-2 (lowest) & -6.7018 & $\mathrm{~S}-7$ & -6.7010 \\
$\mathrm{~S}-3$ & -6.6943 & $\mathrm{~S}-8$ & -6.7016 \\
$\mathrm{~S}-4$ & -6.6976 & $\mathrm{~S}-9$ & -6.6981 \\
$\mathrm{~S}-5$ & -6.7009 & $\mathrm{~S}-10$ & -6.6917 \\
$\beta-\mathrm{B}_{106}$ & -6.7035 & $\alpha-\mathrm{B}_{12}$ & -6.7048 \\
\hline \hline
\end{tabular}




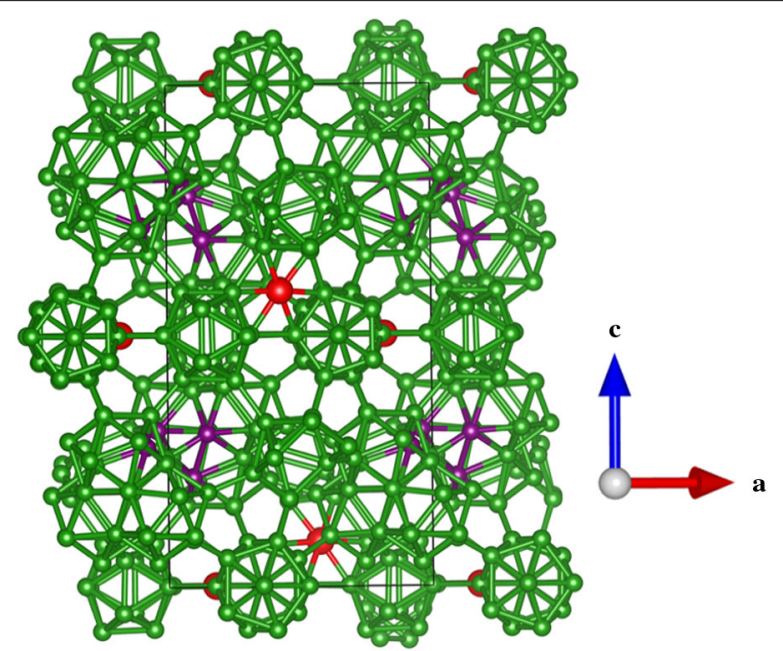

FIG. 1. The lowest energy structure found for the $\tau$ - $\mathrm{B}_{106}$ structure. We considered 10 possible distributions for the partially occupied B13 and B16 sites with the energies in Table I (the structures are in Ref. [8]). The lowest is S2 while the energies of S8 and S7 are only 0.2 and $0.6 \mathrm{meV} / \mathrm{B}$ higher. The lowest energy structure has the same B13 and B16 sites as $\beta$ - $\mathrm{B}_{106}$ although this structure breaks the symmetry along the twin boundary. These B13 sites are shown as purple while the B16 sites are red with all other sites green. The definition of various sites in $\beta$ - $\mathrm{B}_{106}$ can be found in Ref. [10].

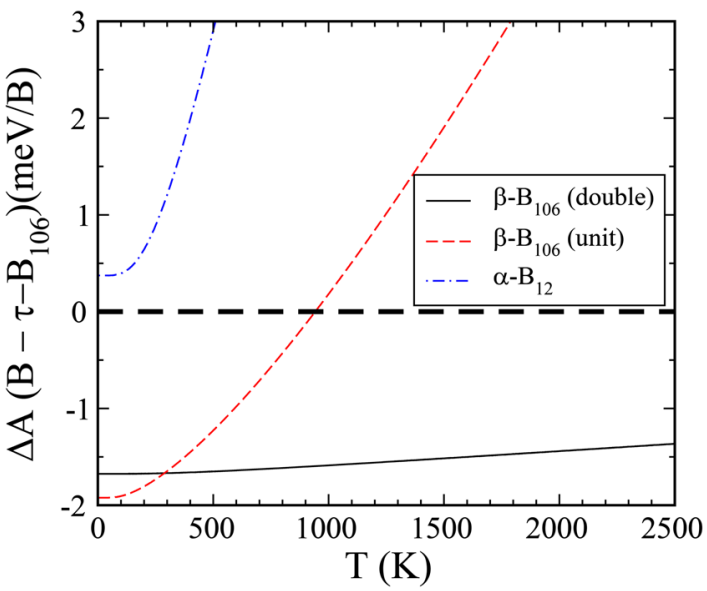

FIG. 2. Helmholtz Free energy differences of $\beta-\mathrm{B}_{106}$ and $\alpha-\mathrm{B}_{12}$ relative to $\tau-\mathrm{B}_{106}$ as a function of temperature [9]. The red dashed curve uses the phonon calculations for the 106 atom crystal unit cell $\beta$ - $\mathrm{B}_{106}$ and the 212 atom crystal unit cell $\tau$ - $\mathrm{B}_{106}$. This indicates that $\tau$ - $\mathrm{B}_{106}$ may be more stable than $\beta$ - $\mathrm{B}_{106}$ at temperatures above $950 \mathrm{~K}$. We consider that comparing the phonon contributions for systems with two different numbers of atoms per cell might bias the corrections because of the particular sampling of the Brillouin zone, so we recalculated the phonon corrections for $\beta-\mathrm{B}_{106}$ using the doubled unit cell (212 atoms). This leads to the solid black line, indicating that the $\beta$ - $\mathrm{B}_{106}$ phase is the most stable phase until the melting temperature of $2350 \mathrm{~K}$.

[1] G. S. Darsavelidze, O. A. Tsagareishvili, T. V. Eterashvili, V. S. Metreveli, G. F. Tavadze, and D. I. Khomeriki, J. Less-Common Met. 117, 189 (1986).

[2] F. N. Tavadze, I. A. Bairamashvili, G. V. Tsagareishvili, K. P. Tsomaya, and N. A. Zoidze, Sov. Phys. Crystallogr. 9, 768 (1965).

[3] T. Ogitsu, V. Lordi, E. Schwegler, and M. Widom, this issue, Phys. Rev. Lett. 118, 159601 (2017).

[4] G. Kresse and J. Hafner, Phys. Rev. B 47, 558 (1993).

[5] G. Kresse and J. Furthmüller, Comput. Mater. Sci. 6, 15 (1996).

[6] G. Kresse and J. Furthmüller, Phys. Rev. B 54, 11169 (1996).

[7] G. Kresse and D. Joubert, Phys. Rev. B 59, 1758 (1999).

[8] The various $\tau$-B106 structures could be found at https://drive.google.com/open?id=0B4BhFV36dCUjU2lqNmtNSEpHdG8.

[9] S. T. Lin, M. Blanco, and W. A. Goddard, III J. Chem. Phys. 119, 11792 (2003).

[10] J. L. Hoard, D. B. Sullenger, C. H. L. Kennard, and R. E. Hughes, J. Solid State Chem. 1, 268 (1970). 